\title{
Human Activity Recognition Using Smart phones
}

\author{
Aman Gupta ${ }^{1}$ | Nidhi Senger ${ }^{2}$ \\ ${ }^{1}$ B.Tech Scholar, Department of IT, Maharaja Agrasen Institute of Technology, Delhi, India \\ ${ }^{2}$ Assistant Professor, Department of IT, Maharaja Agrasen Institute of Technology, Delhi, India
}

To Cite this Article

Aman Gupta and Nidhi Senger, "Human Activity Recognition Using Smart phones ", International Journal for Modern Trends in Science and Technology, 6(12): 445-448, 2020.

\section{Article Info}

Received on 16-November-2020, Revised on 09-December-2020, Accepted on 12-December-2020, Published on 19-December-2020.

\section{ABSTRACT}

Human-centered computing is an emerging research field that aims to understand human behavior and integrate users and their social context with computer systems. One of the most recent, challenging and appealing applications in this framework consists in sensing human body motion using smartphones to gather context infor-mation about people actions. In this context, we describe in this work an Activity Recognition database, built from the recordings of 30 subjects doing Activities of Daily Living (ADL) while carrying a waist-mounted smartphone with embedded inertial sensors, which is released to public domain on a well-known on-line repos-itory. Results, obtained on the dataset by exploiting a multiclass Support Vector Machine (SVM), are also acknowledged.

KEYWORDS: Data science and Machine learning

\section{INTRODUCTION}

Human Activity Recognition (HAR) aims to identify the actions carried out by a person given a set of observations of him/herself and the surrounding environment. Recognition can be accomplished by exploiting the information retrieved from various sources such as environmental or body-worn sensor. Some approaches have adapted dedicated motion sensors in different body parts such as the waist, wrist, chest and thighs achieving good classification performance. These sensors are usually un-comfortable for the common user and do not provide a long-term solution for activity monitoring (e.g. sensor repositioning after dressing ). Smartphones are bringing up new research opportunities for human-centered ap-plications where the user is a rich source of context information and the phone is the firsthand sensing tool. Latest devices come with embedded built-in sensors such as microphones, dual cameras, accelerometers, gyroscopes, etc. The use of smart phones with inertial sensors is an alternative solution for HAR. These mass-marketed devices provide a flexible, affordable and self-contained solution to automatically and unobtru-sively monitor Activities of Daily Living (ADL) while also providing telephony services. Consequently, in the last few years, some works aiming to understand human behavior using smartphones have been proposed: for instance in, one of the first approaches to exploit an Android smartphone for HAR employing its embedded triax-ial accelerometers; additional results have also been presented. Improvements are still expected in topics such as in multi-sensor fusion for better HAR classification, standardizing performance 
evaluation metrics, and providing public data for evaluation.

\begin{tabular}{|r|l|r|}
\hline No. & Static & Time (sec) \\
\hline OStart (Standing & 0 \\
0 & Pos) & 15 \\
1 & Stand (1) & 15 \\
2 & Sit (1) & 15 \\
3 & Stand (2) & 15 \\
4 & Lay Down (1) & 15 \\
5 & Sit (2) & 15 \\
6 & Lay Down (2) & 15 \\
\hline No. dynamic & Time (sec) \\
\hline 7 & Walk (1) & 120 \\
8 & Walk (2) & TOTAL \\
\hline & TOTA
\end{tabular}

Table 1: Protocol of activities for the HAR Experiment.

In the HAR research framework, some datasets have been released to the public do-main: the one of the Opportunity Project is an example which has recorded a set of ADL in a sensor rich environment using 72 environmental and body sensors. Similarly, other works have provided public data.Publicly available datasets provide a freely available source of data across different disciplines and researchers in the field. For this reason, we present a new dataset that has been created using inertial data from smartphone accelerometers and gyroscopes, targeting the recognition of six different human activities. Some results, obtained by exploiting a multi class Support Vector Machine (SVM) classifier, are shown as well.

\section{Methodology}

The experiments have been carried out with a group of 30 volunteers within an age bracket of 19-48 years. Each person performed four activities (WALKING, SITTING, STANDING, LAYING) wearing a smartphone (Samsung Galaxy S II) on the waist. Using its embedded accelerometer and gyroscope, we captured 3-axial linear acceleration and 3-axial angular velocity at a constant rate of $50 \mathrm{~Hz}$. The experiments have been video-recorded to label the data manually. The obtained dataset has been randomly partitioned into two sets, where $70 \%$ of the volunteers was selected for generating the training data and 30\% the test data. The sensor signals (accelerometer and gyroscope) were pre-processed by applying noise filters and then sampled in fixed-width sliding windows of $2.56 \mathrm{sec}$ and $50 \%$ overlap (128 readings/window). The sensor acceleration signal, which has gravitational and body motion components, was separated using a Butterworth low-pass filter into body acceleration and gravity. The gravitational force is assumed to have only low frequency components, therefore a filter with $0.3 \mathrm{~Hz}$ cutoff frequency was used. From each window, a vector of features was obtained by calculating variables from the time and frequency domain.

\section{Signal Processing}

We collected triaxial linear acceleration and angular velocity signals using the phone accelerometer and gyroscope at a sampling rate of $50 \mathrm{~Hz}$. These signals were pre-processed for noise reduction with a median filter and a 3rd order low-pass Butter-worth filter with a $20 \mathrm{~Hz}$ cutoff frequency.

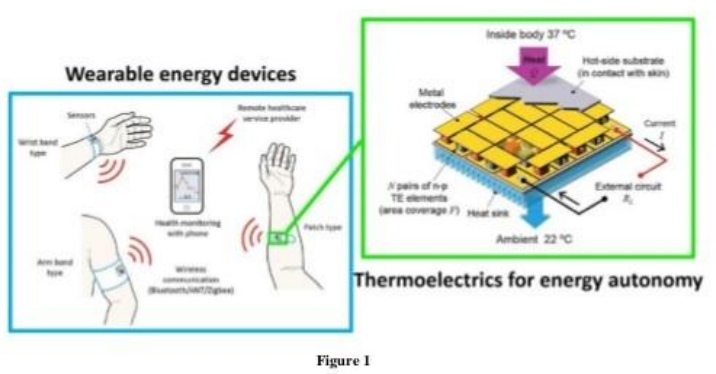

\begin{tabular}{|l|r|r|}
\hline Name & Time & Freq. \\
\hline Body Acc & 1 & 1 \\
Gravity Acc & 1 & 0 \\
Body Acc Jerk & 1 & 1 \\
Body Angular Speed & 1 & 1 \\
Body Angular Acc & 1 & 0 \\
Body Acc Magnitude & 1 & 1 \\
Gravity Acc Mag & 1 & 0 \\
Body Acc Jerk Mag & 1 & 1 \\
Body Angular Speed & & \\
Mag & 1 & 1 \\
Body Angular Acc & 1 & 1 \\
Mag & & \\
\hline
\end{tabular}

Table 2: Time and frequency domain signals obtained from the smartphone sensors.

This rate is sufficient for capturing human body motion since $99 \%$ of its energy is contained below $15 \mathrm{~Hz}$ [3]. The acceleration signal, which has gravitational and body motion components, was separated using an-other Butterworth low-pass filter into body acceleration and gravity. The 
gravitational force is assumed to have only low frequency components, therefore we found from the experiments that $0.3 \mathrm{~Hz}$ was an optimal corner frequency for a constant gravity signal.

Additional time signals were obtained by calculating from the triaxial signals the euclidean magnitude and time derivatives (jerk $d a / d t$ and angular acceleration $d w / d t$ ). The time signals were then sampled in fixed-width sliding windows of $2.56 \mathrm{sec}$ and $50 \%$ overlap between them, since :

- The cadence of an average person walking is within $[90,130]$ steps/min [14], i.e. a minimum of 1.5 steps/sec;

- At least a full walking cycle (two steps) is preferred on each window sample;

- People with slower cadence such as elderly and disabled should also benefit from this method. We supposed a minimum speed equal to $50 \%$ of average human cadence;

- Signals are also mapped in the frequency domain through a Fast Fourier Trans-form (FFT), optimized for power of two vectors $(2.56 \mathrm{sec} \times 50 \mathrm{~Hz}=$ 128 cycles).

Thus, a total of 17 signals were obtained with this method, which are listed in Table 2.

\section{Feature Mapping}

From each sampled window described above a vector of features was obtained. Stan-dard measures previously used in HAR literature [15] such as the mean, correlation, signal magnitude area (SMA) and autoregression coefficients [16] were employed for the feature mapping. A new set of features was also employed in order to improve the learning performance, including energy of different frequency bands, frequency skew-ness, and angle between vectors (e.g. mean body acceleration and $y$ vector). Table 3 contains the list of all the measures applied to the time and frequency domain signals. A total of 561 features were extracted to describe each activity window. In order to ease the performance assessment, the dataset has been also randomly partitioned into two independent sets, where $70 \%$ of the data were selected for training and the remaining $30 \%$ for testing.

\begin{tabular}{|l|l|}
\hline Function & Description \\
\hline mean & Mean value \\
std & Standard deviation \\
mad & Median absolute value \\
max & Largest values in array \\
min & Smallest value in array \\
sma & Signal magnitude area \\
energy & Average sum of the squares \\
iqr & Interquartile range \\
entropy & Signal Entropy \\
arCoeff & Autorregresion coefficients \\
correlation & Correlation coefficient \\
maxFreqIn & Largest frequency \\
d & component \\
& Frequency signal weighted \\
meanFreq & average \\
skewness & Frequency signal Skewness \\
kurtosis & Frequency signal Kurtosis \\
energyBan & Energy of a frequency \\
d & interval \\
angle & Angle between two vectors \\
\hline
\end{tabular}

3: List of measures for computing feature vectors.

The Human Activity Recognition dataset has been made available for public use and it is presented as raw inertial sensors signals and also as feature vec-tors for each pattern. It has been submitted as the Human Activity Recognition using Smartphones dataset in the UCI Machine Learning Repository.

\section{RESULTS}

We conducted some experiments on the HAR dataset to acknowledge future users with some results. For this purpose, we exploit well-known and state-of-the-art Support Vector Machine (SVM) binary classifiers, which are generalized to the multiclass case through a One-Vs-All (OVA) approach: the SVM hyper parameters are selected through a 10-fold Cross Validation procedure and Gaussian kernels are used for our experiments. The classification results using the multiclass SVM (MC-SVM) for the 4 ADL are presented in Table 4. They show an overall accuracy of $96 \%$ for the test data composed of 2947 patterns. Similar work on HAR using special purpose sensors have shown comparable performance $(90 \%-96 \%)$, such as in [3] where a system developed by collecting data from 6 volunteers for the classification of 12 ADL using a waist-mounted triaxial accelerometer provided an accuracy of $90.8 \%$, and similarly in [18] where a chest-mounted accelerometer was used for classifying 5 ADL obtained a recognition performance of $93.9 \%$. This allows to argue that the use of smartphones, in addition to be more unobtrusive and less invasive than other special 
purpose solutions (e.g. wear-able sensors), is a feasible way to walk for effectively performing HAR.

Table 4: Confusion Matrix of the classification results on the test data using the multi-class SVM. Rows represent the actual class and columns the predicted class. Activity names on top are abbreviated.

\begin{tabular}{|l||rrrr|r|}
\hline & WK & ST & SD & LD & Recall \\
\hline Walking & $\mathbf{4 9 2}$ & 0 & 0 & 0 & $99 \%$ \\
Sitting & 0 & $\mathbf{4 3 2}$ & 57 & 0 & $88 \%$ \\
Standing & 0 & 14 & $\mathbf{5 1 8}$ & 0 & $97 \%$ \\
Laying & & 0 & 0 & $\mathbf{5 3 7}$ & $100 \%$ \\
Down & 0 & 0 & 0 & \\
& & & & & \\
& & & & 100 & \\
& & & & \\
& & & \\
& & &
\end{tabular}

\section{DISCUSSION}

In this paper we introduced a new publicly available dataset for HAR using smart phones and acknowledged some results using a multiclass Support Vector Machine approach. The multiclass SVM employed for the classification of smart phone inertial data showed a recognition performance similar to previous work that have used special purpose sensors, therefore strengthening the application of these devices for HAR purposes.

We also highlighted an improvement on the classification performance of the learned model using this new dataset against the previous version, which had a reduced set of features. However, rooms for improvements exist: while dynamic activities can be efficiently classified thanks to the newly introduced features in the released dataset, non-dynamic actions still present misclassification overlaps. This requires further study of available inputs and revision of the HAR process pipeline phases. Finally, computational complexity aspects such as battery life and real time processing for the application will be assessed in our forthcoming works.

\section{REFERENCES}

1. R.Poppe. Vision-based human motion analysis: An overview. Computer Vision and Image Under-standing, 108(1-2):4-18, 2007.
2. J.R. Kwapisz, G.M. Weiss, and S.A. Moore. Activity recognition using cell phone accelerometers. SIGKDD Explorations Newsletter, 12(2):74-82, 2011.

3. A. Frank and A. Asuncion. UCI machine learning repository, 2010.

4. C.Cortes and V.Vapnik. Support-vector networks. Machine learning, 20(3):273-297, 1995.

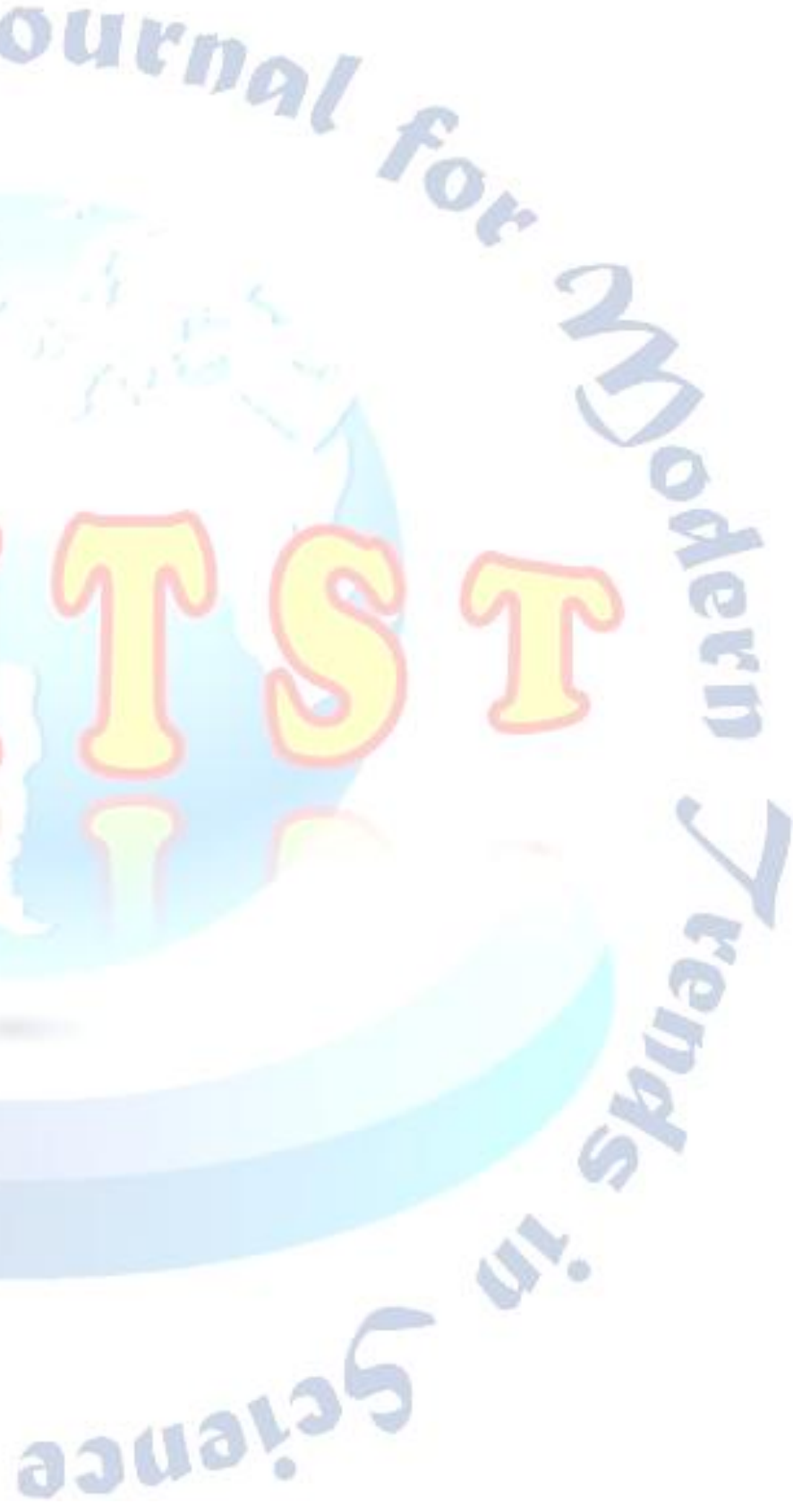

